\title{
Effect of air-abrasion regimens and fine diamond bur grinding on flexural strength, Weibull modulus and phase transformation of zirconium dioxide
}

\author{
Silvia Masae de Araújo Michida ${ }^{1}$, Estevão Tomomitsu Kimpara ${ }^{2}$, Claudinei dos Santos ${ }^{3}$, Rodrigo Othavio Assunção Souza ${ }^{4}$, \\ Marco Antonio Bottino², Mutlu Özcan ${ }^{5}$ \\ ${ }^{1}$ Ingá University, Dental School, Post Graduate Program, Maringa - Brazil \\ ${ }^{2}$ São Paulo State University, São José dos Campos Dental School, Department of Dental Materials and Prosthodontics, São José dos Campos - Brazil \\ ${ }^{3}$ Rio de Janeiro State University, Department of Mechanics and Energy, Technology Faculty, Resende - Brazil \\ ${ }^{4}$ Federal University of Rio Grande do Norte (UFRN), Department of Restorative Dentistry, Division of Prosthodontics, Natal - Brazil \\ ${ }^{5}$ University of Zurich, Dental Materials Unit, Center for Dental and Oral Medicine, Clinic for Fixed and Removable Prosthodontics and Dental \\ Materials Science, Zürich - Switzerland
}

\begin{abstract}
Purpose: This study evaluated the effect of air abrasion and polishing regimens on the flexural strength of yttrium stabilized polycrystalline tetragonal zirconia (Y-TZP).

Methods: From Y-TZP blocks (InCeram 2000 YZ Cubes; Vita Zahnfabrik, Bad Säckingen, Germany) 120 bars (25 mm × $4 \mathrm{~mm} \times 1.2 \mathrm{~mm}$ ) were obtained according to ISO 6872:2008 and randomly divided into 4 groups: Group C: (control) without surface treatment $(n=30)$; Group APA: Air abrasion with aluminum oxide $(44 \mu \mathrm{m})(n=30)$; Group SC: Silicacoating (CoJet, $30 \mu \mathrm{m})(n=30)$; Group FD: Fine diamond bur $(n=30)$. Subsequently, all specimens were subjected to 4-point bending test (in distilled water at $37^{\circ} \mathrm{C}$ ) in a universal testing machine (EMIC DL 1000; São José dos Pinhais, Paraná, Brazil); cross-head speed: $0.5 \mathrm{~mm} / \mathrm{min}$ ). The characteristic strength $\left(\sigma_{0}\right)$ of each specimen was obtained from the flexural strength test and evaluated using Weibull analysis. X-ray diffraction analysis was utilized to quantity the monoclinic phase. The surface topography of specimens was analyzed using 3D optical profilometer and scanning electron microscopy (SEM) after surface conditioning methods. The flexural strength data $\left(\sigma_{4 \mathrm{p}}\right)$ were statistically analyzed by 1-way ANOVA, Tukey test $(\alpha=0.05)$ and Weibull ( $\mathrm{m}=$ modulus, $\sigma_{0}=$ characteristic strength) were calculated. Results: The mean \pm standard deviations (MPa) of the groups were as follows: C: $1196.2 \pm 284.2^{\mathrm{a}}$; APA: $1369.7 \pm$ $272.3^{\mathrm{a}}$; SC: $1207.1 \pm 229.7^{\mathrm{a}}$ and FD: $874.4 \pm 365.4^{\mathrm{b}}$. The values $(\mathrm{m})$ and $\left(\sigma_{0}\right)$ were as follows: C: 4.5 and 1308.12 ; APA: 5.9 and 1477.88; SC: 6.0 and 1300.28; and FD: 2.6 and 985.901 , respectively.

Conclusions: Air particle abrasion with neither silica nor alumina showed significant difference compared to the control group but grinding with fine diamond bur impaired the flexural strength of the zirconia tested.

Keywords: Air abrasion, Polishing, Flexural strength, Weibull modulus, Phase transformation, Yttria-stabilized tetragonal zirconia
\end{abstract}

\section{Introduction}

Zirconia is a densely sintered ceramic that offers chemically stable restorations with improved aesthetics. Yttria-stabilized

Accepted: February 25, 2015

Published online: September 18, 2015

Corresponding author:

Prof. Dr. med. dent. Mutlu Özcan, PhD

Head of Dental Materials Unit

University of Zürich

Center for Dental and Oral Medicine

Clinic for Fixed and Removable Prosthodontics

and Dental Materials Science

Plattenstrasse 11

$\mathrm{CH}-8032$ Zürich, Switzerland

mutluozcan@hotmail.com tetragonal zirconia polycrystal (Y-TZP) offers good physical properties, including high flexural strength and high fracture toughness compared to other ceramic materials (1). In addition to favorable mechanical properties, chemical and dimensional stability (2) zirconia frameworks exhibit good radiopacity, enhancing radiographic evaluation of marginal integrity and detection of recurrent decay (3).

Initially, conventional cements such as zinc phosphate or resin-modified glass ionomer cements were suggested for luting zirconia reconstructions. However, adhesive cementation has been shown to increase fracture resistance and improve longevity of ceramic restorations $(4,5)$. In addition, resin cements offer the advantage of sealing minor internal surface flaws created by acid etching or airborne particle abrasion, which significantly strengthens ceramic materials (6). Zirconia restorations present an arbitrary cement space incorporated in the coping design, resulting in a loose fit. Furthermore, 
auxiliary retentive designs commonly used in metal ceramic restorations are not routinely incorporated in zirconia restorations. The minimal inherent mechanical retention in CAD/ CAM-milled zirconia restorations indicates the need for improved adhesion. A reliable bonding protocol for zirconia ceramics would further expand the minimal invasive applications of this new- generation aesthetic reconstruction material. Resin bonding to silica-based ceramics could be best achieved using surface conditioning methods based on mechanical roughening or chemical activation of the ceramic surface. Unfortunately, zirconia surface is minimally affected by conventional surface roughening methods (7). Furthermore, the relative inertness of zirconia renders the surface unreactive to adhesive promoters.

Various methods have been investigated in an attempt to roughen and/or activate the zirconia surface to improve adhesion. Previous reports suggest that mechanical roughening procedures may enhance the strength of zirconia ceramics (8-13). Airborne-particle abrasion, which is the method of choice for roughening the intaglio surface of conventional metal-ceramic restorations prior to cementation, has been contraindicated for feldspathic ceramic restorations due to the high abrasion rate and subsequent volume loss (14). Yet, the effect of air abrasion on the fracture strength of high-strength ceramic materials has been discussed controversially in the literature. Some reports advocate air abrasion of zirconia restorations to enhance their strength $(8,9)$ whereas others present concerns about the potential long-term adverse effect of surface abrasion on the strength of zirconia $(15,16)$. Wet hand-grinding with a finegrit diamond rotary cutting instrument is another surface modification described in the literature, with a favourable effect on the strength of zirconia $(11,17)$. Previous investigators advocated fine grinding as an alternative method for roughening zirconia reconstructions in the absence of air abrasion armamentarium $(10,12,18)$.

Tribochemical silicoating has been introduced as an alternative to air abrasion with alumina particles in order to improve the adhesion between metals or oxide-based ceramics and resin cements (14). In this technique, the surfaces are airborne-particle abraded with aluminum trioxide particles modified with silica. The blasting pressure results in the embedding of the silica-coated alumina particles on the ceramic surface, rendering the silica-modified surface chemically reactive to the resin through silane coupling agents (19). To date, there is no consensus regarding the best surface conditioning method for achieving optimum bond strength to zirconia. The published data on the short- and long-term resin bond strength to zirconia following different surface conditioning regimens show diverse results (13). There are also concerns that the surface of the metastable tetragonal zirconia ceramic will be transformed, i.e. constrained, and also damaged that will eventually influence the mechanical properties and reliability of the material.

The objective of this study therefore was to evaluate the effect of air abrasion and polishing regimens on the flexural strength of Y-TZP ceramic. The hypothesis tested was that mechanical surface modification of the zirconia for luting procedures would affect its flexural strength and its phase transformation.

\section{Materials and methods}

\section{Specimen preparation}

Bars $(\mathrm{N}=120)(5 \mathrm{~mm} \times 1.6 \mathrm{~mm} \times 25 \mathrm{~mm})$ were milled from partially sintered 5\% Y-TZP (InCeram 2000 YZ Cubes YZ-40/19; Vita Zahnfabrik, Bad Säckingen, Germany) using an electrical high-precision saw (Labcut 1010; Erios, São Paulo, Brazil) under water irrigation with a diamond disc. They were then sintered at $1500^{\circ} \mathrm{C}$ for $7 \mathrm{~h}$ in a high temperature sintering furnace assigned for zirconia (Vita Zyrcomat T, Vita Zahnfabrik). The dimensions of the bars following $22 \%$ volumetric shrinkage associated with the sintering were $4 \mathrm{~mm} \times 1.2 \mathrm{~mm} \times 20 \mathrm{~mm}$ $( \pm 0.2 \mathrm{~mm}, \pm 0.2 \mathrm{~mm}$, and $\pm 0.3 \mathrm{~mm}$, respectively). The specimens were randomly assigned to 4 groups ( $n=30$ per group):

Control group (C): This group underwent no mechanical surface conditioning method following sintering.

Air-borne particle abrasion (APA): The bars were airabraded using $44 \mu \mathrm{m} \mathrm{Al}_{2} \mathrm{O}_{3}$ particles (Polidental, Cotia, São Paulo, Brazil) at 2.8 bar pressure from a distance of $10 \mathrm{~mm}$ perpendicular to the long axis of the bars for 10 seconds.

Silicacoating (SC): The bars in this group were airborneparticle abraded using a chairside air abrasion device (MicroEtcher, Danville, California, USA) with $30 \mu \mathrm{m}$ silica modified $\mathrm{Al}_{2} \mathrm{O}_{3}$ particles (CoJet, 3M ESPE, St. Paul, Minn) at $2.8 \mathrm{bar}$ pressure for 10 seconds.

Fine-grit diamond (FD): The bars were initially wet ground and finished using a fine-grit diamond rotary cutting instrument (yellow stripe round-end straight diamond, 30- $\mu \mathrm{m}$ grit size; KG Sorensen, Cotia, Brazil) in a high-speed handpiece under water irrigation for $10 \mathrm{~s}$.

\section{Flexural strength test}

Each bar was oriented in a holding jig with the modified surface subjected to tensile forces. The bars were loaded to failure using the 4-bending test in a Universal Testing Machine (EMIC DL 1000, São José dos Pinhais, Paraná, Brazil) with a $100 \mathrm{kgf} \mathrm{load} \mathrm{cell} \mathrm{at} \mathrm{a} \mathrm{cross-head} \mathrm{speed} \mathrm{of} 0.5 \mathrm{~mm} / \mathrm{min}$ (Fig. 1). The tests were performed in a thermostatically controlled water bath at $37^{\circ} \mathrm{C}$.

\section{Statistical analysis}

Statistical analysis was performed with SPSS Statistics for Windows Version 20 (SPSS, Chicago, IL, USA). Shapiro Wilk test indicated normal distribution of the data. Flexural strength data $(\mathrm{MPa})$ were analyzed using 1-way analysis of variance (ANOVA). Multiple comparisons were made using Tukey test $(\alpha=0.05)$. Weibull analysis was performed using Minitab 14 (Minitab, State College, PA, USA). The statistical variability of estimates for the 2 characteristic Weibull parameters $m$ and $\sigma_{0}$ were calculated according to Ritter et al (20). Accordingly, Weibull modulus $m$ depends only on the sample size, i.e., upon the number of specimens per condition, whereas the variability in the characteristic strength $\sigma_{0}$ depends on both sample size and $m$ :

$$
\ln \ln \frac{1}{1-F_{\left(\sigma_{c}\right)}}=m \ln \sigma_{c}-m \ln \sigma_{0}
$$




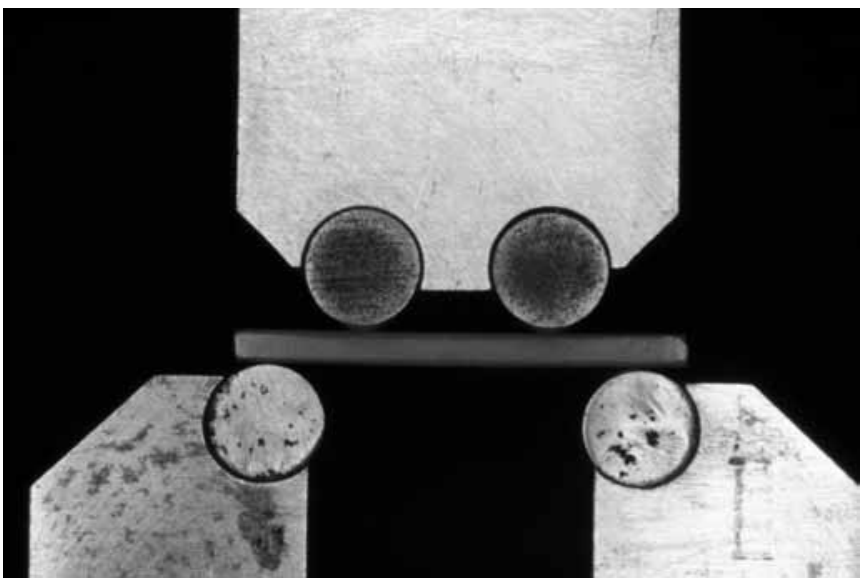

Fig. 1 - Zirconia bar specimen on 4-point bending test.

\section{$X-R a y$ diffraction analysis (XRD)}

The specimens ( $N=8, n=2$ per group) were analyzed in an X-ray diffractometer (D45000; Siemens, Munich, Germany) using monochromatic Cu-K alpha radiation $(\lambda=1.54060 \AA$ ). Scans were performed at $40 \mathrm{kV}, 40 \mathrm{~mA}, 0.02^{\circ} / \mathrm{step}$, with step interval ranging from $20^{\circ}$ to $60^{\circ}$, at $1 \mathrm{~s}$ per step.

The relative amount of transformed monoclinic zirconia $\left(F_{M}\right)(\%)$ on the conditioned surfaces was determined from the integral intensities of the monoclinic (-111)M and (111)M, and the tetragonal (101)T peaks obtained using XRD, according to the method described by Toraya et al (21) using the equations ( $A$ and $B$ ) below:

$$
\begin{gathered}
F_{M}=\frac{1.311 * X_{M}}{1+0.311^{*} X_{M}} \\
X_{M}=\frac{(-111)_{M}+(111)_{M}}{(-111)_{M}+(111)_{M}+(101)_{T}}
\end{gathered}
$$

where $(-111) \mathrm{M}, 2 \theta=28^{\circ} ;(111) \mathrm{M}, 2 \theta=31.20 ;(101) \mathrm{T}$, $2 \theta=30^{\circ}$, represent the integrated intensity of the peaks diffracted in the monoclinic planes (-111)M and (111)M and in the tetragonal plane (101)T.

Two measurements were carried out on each specimen to obtain the mean values of $F_{M}$. Raman spectroscopy and
XRD measurements were performed on the tensile side of the specimens.

\section{Transformed zone depth (TZD)}

The transformed zone depth (TZD) $(\mu \mathrm{m})$ on the conditioned surfaces of zirconia was calculated from the relative amounts of the monoclinic phase, assuming that, within the transformed surface layer, all the tetragonal grains have transformed into the monoclinic symmetry, according to the Equation C below (17):

$$
T Z D=\left(\frac{\operatorname{sen} \theta}{2 \mu}\right)\left[\ln \left(\frac{1}{1-F M}\right)\right]
$$

where, $\theta=15^{\circ}$ is the angle of reflection; $\mu(=0.0642)$ is the absorption coefficient and $F_{M}$ the relative monoclinic fraction obtained from the Raman analysis on the basis of equations $A$ and $B$.

\section{D optical profilometer and SEM analyses}

Specimen surfaces were further analyzed using a 3D optical profilometer (Wyko, Model NT 1100; Veeco, Plainview, NY, USA) connected to the software (Wyko Vision 32; Veeco, USA) for topography data analysis from a total area of $0.06 \mathrm{~mm}^{2}$ at $\times 20$ magnification.

The microstructure and surface topography of the specimens were analyzed using SEM (LEO-32, Model 1450 VP; Carl Zeiss Nano Technology Stystems, Welwyn Garden City, UK) at $\times 3000$ magnification after gold sputtering (MED 020 Coating System; Bal-Tec, Berkeley, CA).

\section{Results}

Mean flexural strength values (MPa) and standard deviation for each group are listed in Table I. Significant difference was observed between the groups (ANOVA, $P=0.00001$ ). Group FD showed the lowest flexural strength results (874.4 $\pm 365.4)^{\mathrm{B}}$ among all groups (1196.2 $\left.\pm 284.2-1369.7 \pm 272.3\right)$ (Tukey test). Air abrasion regimens using either alumina $(1369.7 \pm 272.3)^{\mathrm{A}}$ or silica $(1207.1 \pm 229.7)^{\mathrm{A}}$ did not show significant difference between one another and the control group $(1196.2 \pm 284.2)^{A}$.

Weibull statistics of the flexural strength test including the characteristic strength, $\sigma_{0}$ (the scale parameter) and the Weibull modulus, $\mathrm{m}$ (the slope parameter) are summarized

TABLE I - Mean ( \pm SD) of flexural strength (MPA) according to surface conditioning methods

\begin{tabular}{lcccccc}
\hline Surface conditioning & $\mathbf{n}$ & Mean & $\begin{array}{c}\text { Standard } \\
\text { deviation }\end{array}$ & $\begin{array}{c}\text { Coefficient of } \\
\text { variation }\end{array}$ & Minimum & Maximum \\
\hline C & 30 & $1196.2^{\mathrm{a}}$ & 284.2 & 23.75 & 789.5 & 1791.7 \\
APA & 30 & $1369.7^{\mathrm{a}}$ & 272.3 & 19.88 & 774.1 & 1846.4 \\
SC & 30 & $1207.1^{\mathrm{a}}$ & 229.7 & 19.03 & 811.8 & 272.5 \\
FD & 30 & $874.4^{\mathrm{b}}$ & 365.4 & 41.79 & 1636.0 \\
\hline
\end{tabular}

$\mathrm{C}=$ control; $\mathrm{APA}=$ air abrasion with alumina particles; $\mathrm{SC}=$ air abrasion with silica particles; FD = fine diamond grinding. 
TABLE II - Weibull statistics of the flexural strength test

\begin{tabular}{cccccccc}
\hline \multicolumn{2}{c}{$\mathbf{C}$} & \multicolumn{2}{c}{ APA } & \multicolumn{2}{c}{ SC } & \multicolumn{2}{c}{ FD } \\
\hline $\mathbf{m}$ & $\boldsymbol{\sigma}_{\mathbf{0}}$ & $\mathbf{m}$ & $\boldsymbol{\sigma}_{\mathbf{0}}$ & $\mathbf{m}$ & $\boldsymbol{\sigma}_{\mathbf{0}}$ & $\mathbf{m}$ & $\boldsymbol{\sigma}_{\mathbf{0}}$ \\
\hline 4.6 & 1308.1 & 6 & 1477.88 & 6.1 & 1300.3 & 2.7 & 985.9 \\
$\sigma_{1 \%}$ & $\sigma_{5 \%}$ & $\sigma_{1 \%}$ & $\sigma_{5 \%}$ & $\sigma_{1 \%}$ & $\sigma_{5 \%}$ & $\sigma_{1 \%}$ & $\sigma_{5 \%}$ \\
479.3 & 684.1 & 685.2 & 899.7 & 607.8 & 795.8 & 174.9 & 322.7 \\
\hline
\end{tabular}

Characteristic strength: $\sigma_{0}$ (the scale parameter), Weibull modulus: $m$ (the slope parameter).

For group abbreviations see Table I.

TABLE III - Relative amounts of monoclinical zirconia and the corresponding TZD values for the experimental groups

\begin{tabular}{lcc}
\hline Groups & $\begin{array}{c}\text { Monoclinic } \\
\text { phase (\%) }\end{array}$ & $\begin{array}{c}\text { Transformed zone } \\
\text { depth }(\text { TZD }(\boldsymbol{\mu m})\end{array}$ \\
\hline C & 0 & 0 \\
APA & 14.46 & 0.79 \\
SC & 12.32 & 0.66 \\
FD & 12.96 & 0.70 \\
\hline
\end{tabular}

in Table II. The highest m-value was obtained with the APA and SC groups, followed by the $C$ group. The FD procedure decreased the $\mathrm{m}$-value about one-half of the $\mathrm{C}$ group.

The highest amount of the monoclinical phase and TZD was found after APA and FD procedures followed by SC (Tab. III).

XRD analysis of sintered Y-TZP ceramics indicate that only group $C$ group consisted of $100 \%$ tetragonal zirconia. After surface conditioning however, detectable monoclinic peaks with a marked preference of the $\mathrm{M}\left(111^{-}\right)$orientations appeared in the XRD pattern (Figs. 2A-D).

SEM and profilometer results presented a certain roughness including the group $\mathrm{C}(0.897 \mu \mathrm{m})$ that was received assintered, without any polishing of the surface after sintering process (Figs. 3AB). Both APA $(0.829 \mu \mathrm{m})$ (Figs. 4AB) and SC $(0.651 \mu \mathrm{m})$ groups (Figs. 5AB) showed less roughness than that of group FD group (0.934 $\mu \mathrm{m})$ (Figs. 6AB).

\section{Discussion}

This study was undertaken in order to investigate the effect of air abrasion and polishing regimens on the flexural strength of Y-TZP. Airborne particle abrasion and silica coating of zirconia resulted in higher flexural strength when compared to the control. Yet, the results were not significant. On the other hand, fine diamond grinding decreased the flexural strength significantly. Thus, the hypothesis that mechanical surface modification of the zirconia surface would affect its flexural strength could be partially accepted.

Kosmac et al (11) demonstrated a similar effect of APA on the flexural strength of zirconia, where tetragonal to monoclinic (T-to-M) phase transformation was induced on the $\mathrm{Y}$ TZP surface. Also, surface grinding has been suggested to create a region of compressive stresses on the Y-TZP surface, which increased its mean flexural strength $(8,9)$. Swain et al (18) demonstrated that hand grinding, when compared to machine grinding, was more effective at inducing the T-to-M phase transformation, thus increasing the strength of zirconia. In another study, Xu et al (10) reported an improvement in the strength of Y-TZP from fine grinding with a $25-\mu \mathrm{m}$ diamond wheel, whereas coarser grinding resulted in strength reduction. Similarly, Kosmac et al (11) advocated fine grinding as a finishing procedure to improve the mean strength and reliability of milled Y-TZP. The results of the current investigation are not in agreement with these previously described reports, since fine diamond grinding significantly decreased the flexural strength of Y-TZP in spite of the compressive stress created.

The use of $30-\mu \mathrm{m}$ silica modified aluminum trioxide particles at 2.8 bar pressure in this study did not result in statistically higher flexure strength compared to APA. Xible et al (22) reported a strengthening effect of tribochemical airborne-particle abrasion using a larger particle size (Rocatec, $110 \mu \mathrm{m} ; 3 \mathrm{M}$ ESPE) at 2.8 bar pressure. The smaller particle size used in the present study was probably less effective at inducing the T-to$M$ phase transformation on the surface of the Y-TZP. The lower flexural strength values, compared to those reported by the manufacturers of Y-TZP, may be due the fact that specimens were only sintered, rather than sintered and polished as recommended by the ANSI/ADA specification for dental ceramics (23). Furthermore, Swain and Hannink (18) reported that hand-ground Ce-TZP surface contained almost 5 times more monoclinic zirconia than severely machine-ground surface of the same material. This was attributed to the extensive heat generated during severe machine grinding in spite of a stream of coolant that was directed near the cutting edge during grinding. Consequently, locally developed temperatures exceeded the temperature for M-to-T transformation, thus the reverse $\mathrm{M}$-to-T transformation occurred. In contrast, the T-to$M$ transformation was retained when hand grinding at lower speed and grinding force, which was not associated with extensive heat generation. Instead, due to high stresses developed during grinding, severe surface cracks must have been formed which decreased the strength and reliability of the material.

Air abrasion, in contrast, is considered to be a more gentle process, during which considerably less material is removed from the surface. In spite of lower stresses occurring during air abrasion, the thickness of the transformed surface layer was found to be larger than in ground specimens, indicating that not only stresses, but also the locally developed temperature during air abrasion was minor. The calculated transformed zone depth, roughly corresponding to the average grain size of sintered Y-TZP ceramic, implies that only those tetragonal zirconia grains have transformed into the monoclinic symmetry during air abrasion that forms the very surface layer. Since these grains are not hydrostatically constrained as those in the bulk of the material, they can readily transform under the stresses caused by deposition impact of the alumina particles. Although the thickness of the surface compressive layer formed during air abrasion is very small in relation to the thickness of the test specimens, it was effective in increasing the strength of the Y-TZP materials. The length of surface flaws that are introduced by air abrasion, does not 

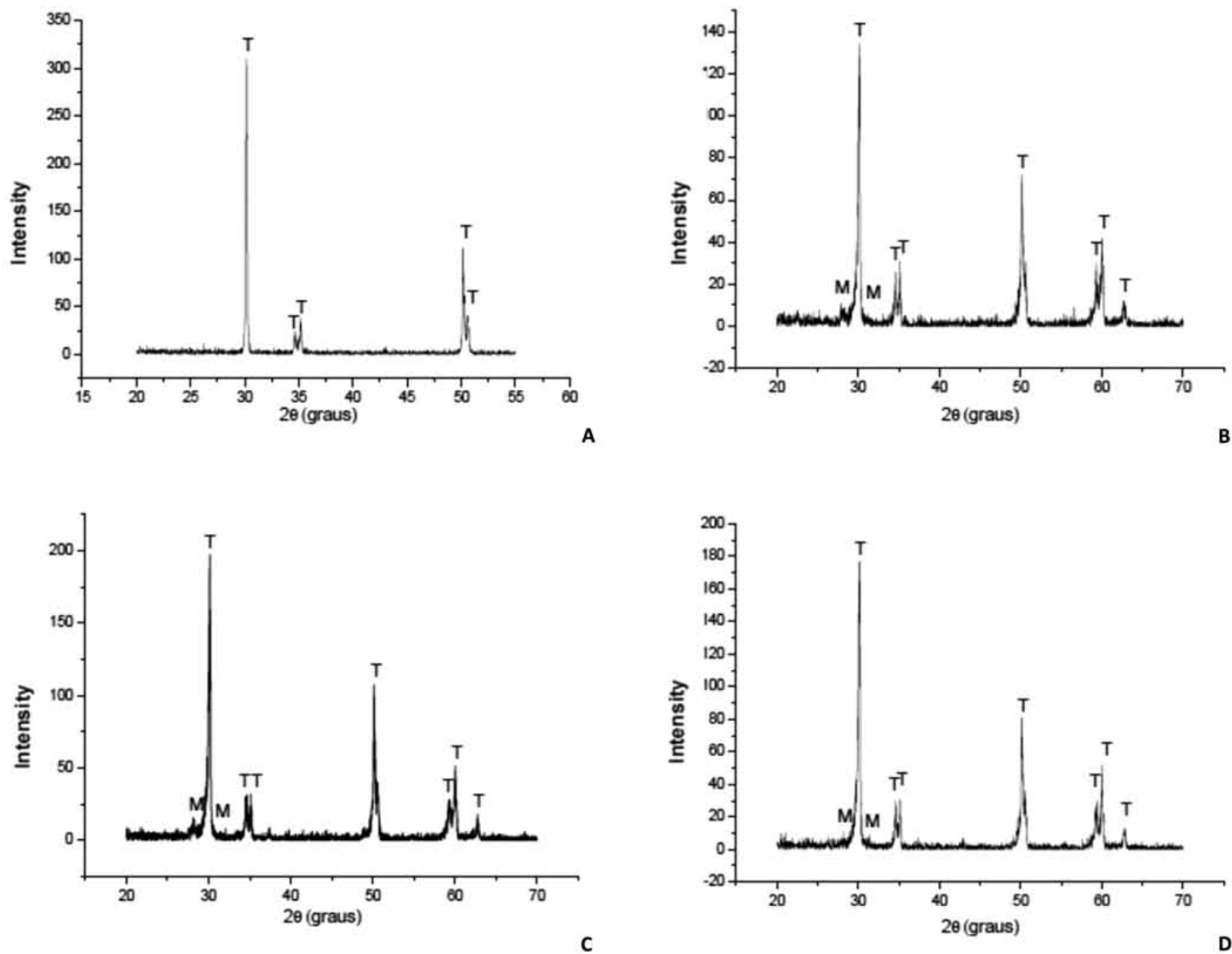

Fig. 2 - XRD analysis of zirconia specimens from groups. (A) Control; (B) Air abrasion with alumina particles; (C) Air abrasion with silica particles; (D) Fine diamond grinding. (T) Tetragonal zirconia phase; (M) monoclinic phase. Note that Control group shows tetragonal phase only.
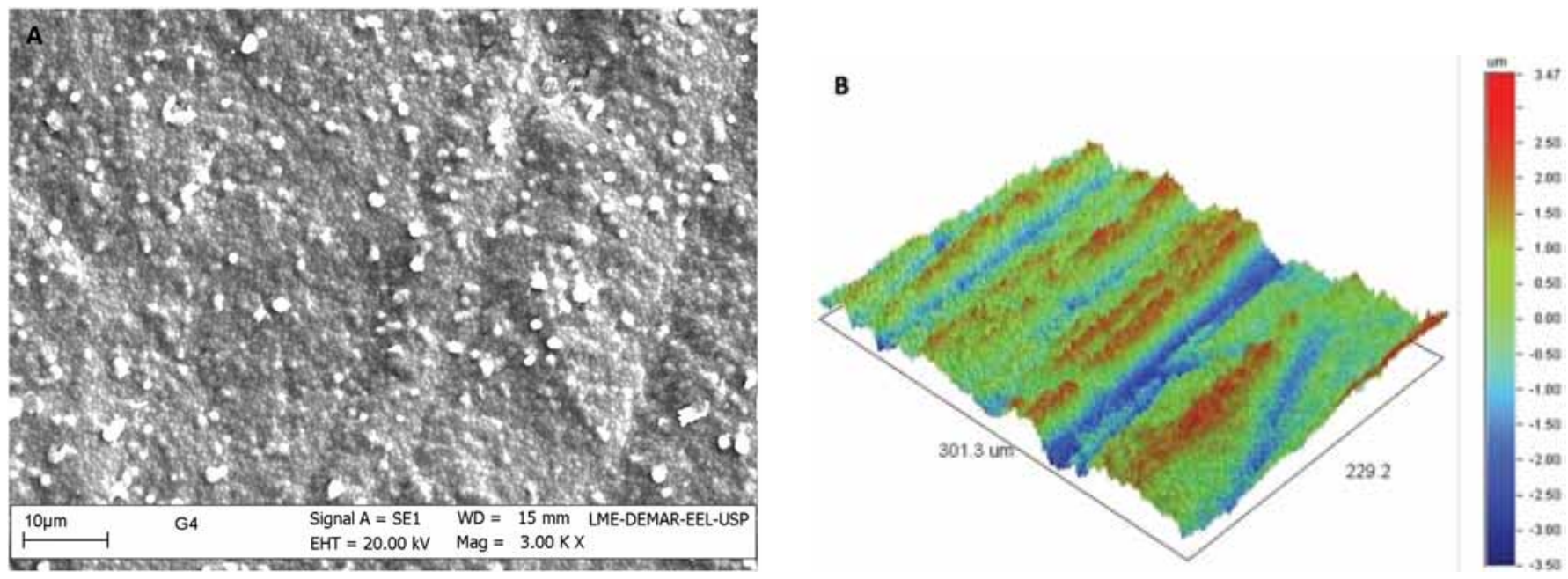

Fig. 3 - (A) Topography of the specimen from the Control group before surface conditioning. Note the rough surface of an as-sintered specimen without any polishing; (B) 3D profilometer image of the surface roughness with mean roughness of $0.897 \mu \mathrm{m}$. 

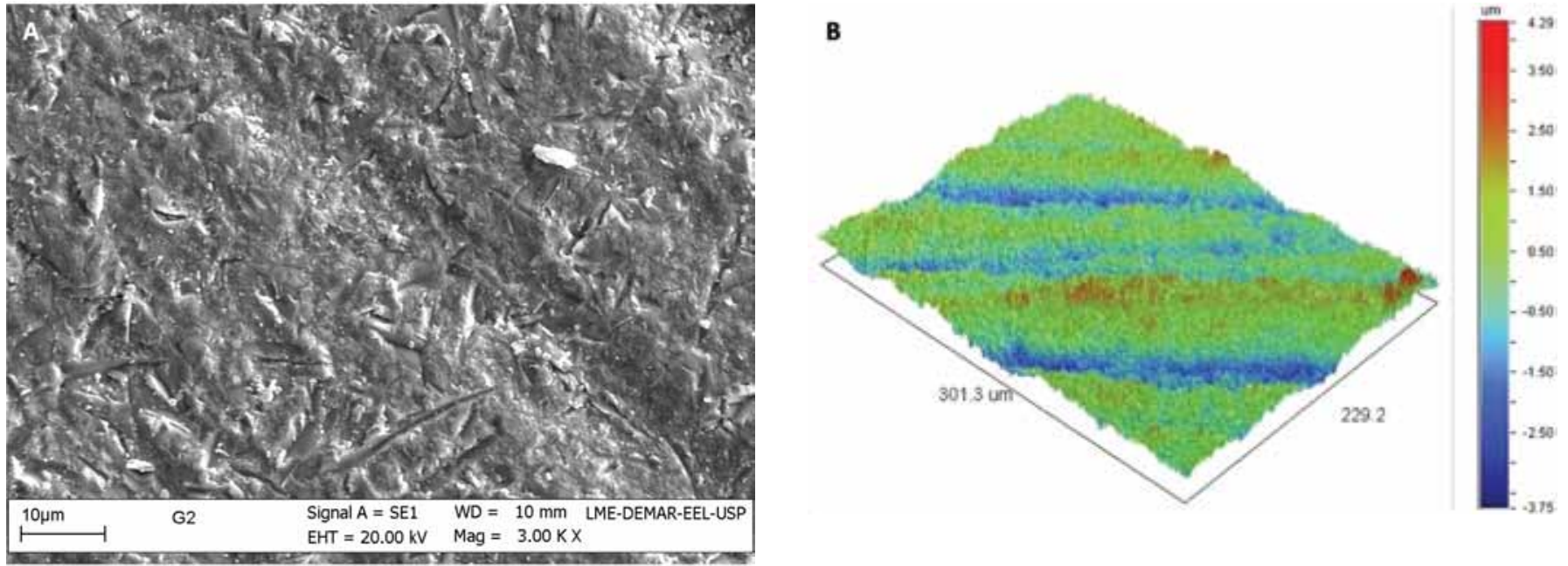

Fig. 4 - (A) Topography of the specimen from the APA group after surface conditioning; (B) 3D profilometer image of the surface roughness with mean roughness of $0.829 \mu \mathrm{m}$.
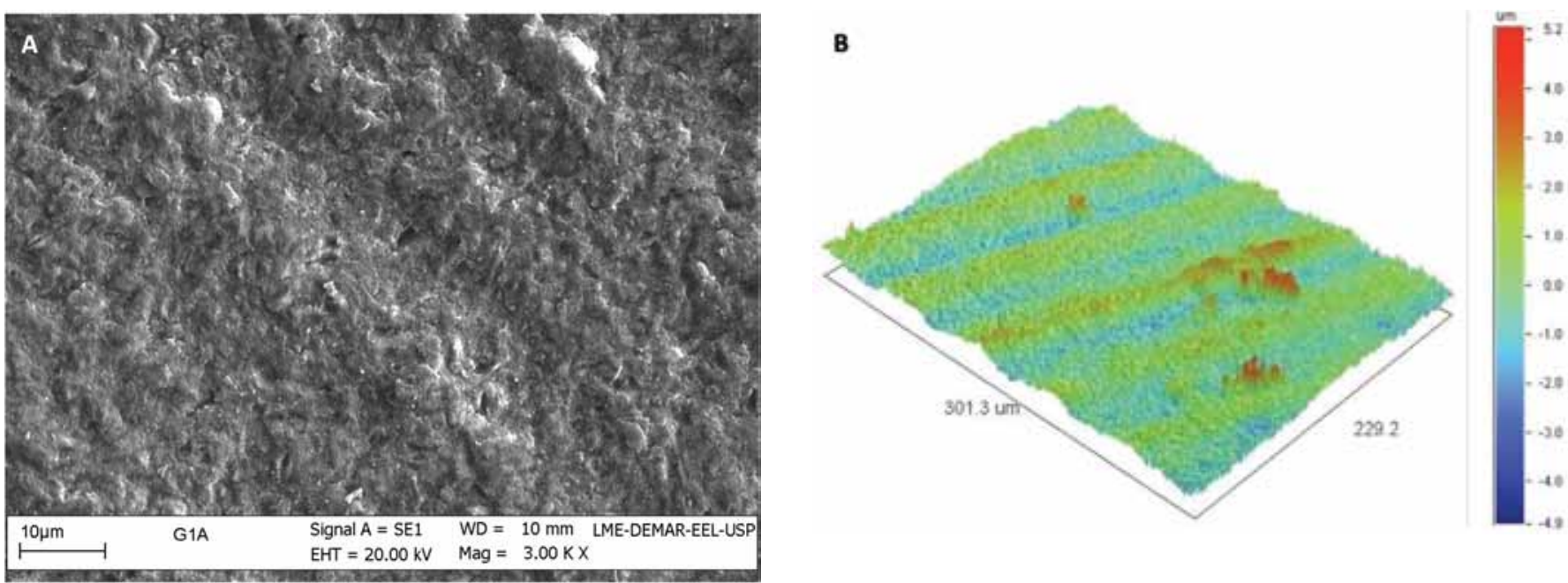

Fig. 5 - (A) Topography of the specimen from the SC group after surface conditioning. Note less roughness intensity compared to other groups; (B) 3D profilometer image of the surface roughness with mean roughness of $0.651 \mu \mathrm{m}$.

seem to exceed largely the thickness of the compressive surface layer, otherwise the strength of the material would have been reduced instead of being increased.

In the study of Souza et al (24), similar particles to those used in this study as well as larger particles of $110 \mu \mathrm{m} \mathrm{Al} \mathrm{O}_{3}$ and $\mathrm{SiO}_{2}$ were used but the pressure was set at 2.5 and 3.5 bar. Higher pressure and particle size resulted in up to $1.43 \mu \mathrm{m}$ TZD and $27.21 \% \mathrm{M}$ phase that are above the results obtained in this study. In a recent study by Özcan et al (25), particles similar to the ones in this study were used but the duration of deposition was $10 \mathrm{~s}$ longer. As expected, up to $1.07 \mu \mathrm{m}$ TZD and $19.25 \%$ $\mathrm{M}$ phase were observed, which are also higher than the results of this study. All these studies imply possible effects of deposition parameters on the transformation and surface morphology changes.

Higher surface roughness values were found in the FD group. In contrast, APA and SC demonstrated less roughness.
This can be explained by the fact that force during particle deposition could erode the peaks and the depths but at the same time they are partially embedded on the surface, eventually diminishing the surface roughness in these groups. Although alumina promotes rougher surface than silica coating, the bond strength between ceramic and resin cement is higher for silica coating than alumina abrasion, because of its chemical interaction to resin cement after silanization (26).

The toughness of Y-TZP ceramic is related to its capacity for tolerating damage and is mostly related to phase transformation, where the $T$ phase is transformed into the $M$ phase. In this transformation, the energy absorbed by the zirconia matrix in the vicinity of the propagating crack is consumed by the $\mathrm{T}$ grains to transform into a $\mathrm{M}$ symmetry, which is accompanied by $\sim 3 \%$ to $4 \%$ volume expansion. This volume expansion hinders crack propagation by means of 

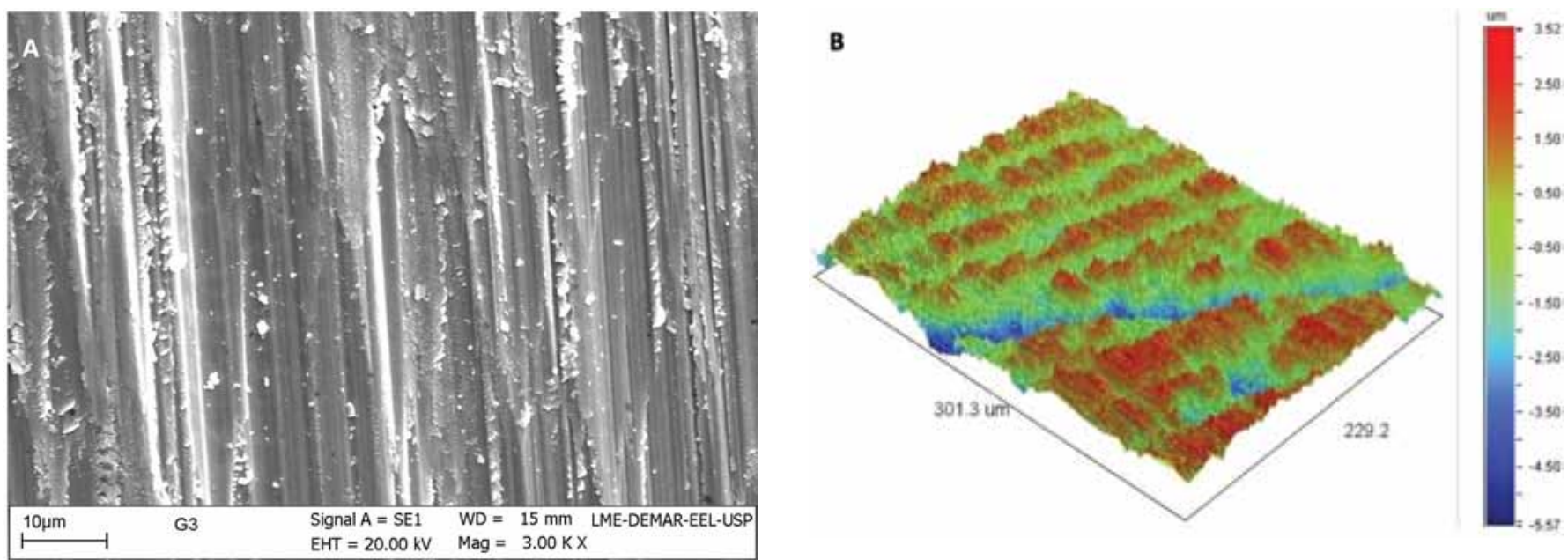

Fig. 6 - (A) Topography of the specimen from the FD group after surface conditioning. Note the high roughness intensity compared to other groups; (B) 3D profilometer image of the surface roughness with mean roughness of $0.651 \mu \mathrm{m}$.

compressive stress $(11,27)$. Thus, in order to maintain this beneficial effect of the Y-TZP ceramic, the phase transformation should be avoided at any rate to avoid crack propagation. The phase transformation in Y-TZP accompanied with the progressive nucleation of $\mathrm{M}$ phase, demonstrated previously $(28,29)$, has a short-term strengthening effect for the compressive stress accumulated on the surface layer. However, the progress of the transformation leads to grain pullout and surface degradation from the applied stresses, leading eventually to the failure of the device enhanced by the aqueous environment. In this study, only static loading was studied, which could be considered a limitation. Dynamic loading could better represent intraoral loading conditions. The long-term effect of mechanical surface modifications of Y-TZP on its flexural strength needs further evaluation in fatigue conditions.

\section{Conclusions}

Considering the flexural strength results, lower Weibull modulus, the higher amount of monoclinic phase, and rougher surface compared to the air-abraded groups and the control group, mechanical surface modification of Y-TZP surface with fine diamond bur cannot be recommended prior to clinical procedures.

\section{Acknowledgement}

The authors gratefully acknowledge Ivan Balducci (São Paulo State University, São José dos Campos Dental School, Department of Social Dentistry and Children Clinic, São José dos Campos, Brazil) for his assistance with the statistical analysis.

\section{Disclosures}

Financial support: The study was supported entirely by Sao Paolo State University.

Conflict of interest: The authors do not have any commercial interest in any of the materials used in this study.
Meeting presentations: Part of this study was presented at the $27^{\text {th }}$ Annual Meeting of the Brazilian Society for Dental Research (SBPqO), September 9 to 12, 2010, Águas de Lindóia, São Paulo, Brazil.

\section{References}

1. Evans AG. Perspective on the development of high-toughness ceramics. J Am Ceram Soc. 1990;73(2):187-206.

2. Aboushelib MN, de Jager N, Kleverlaan CJ, Feilzer AJ. Microtensile bond strength of different components of core veneered all-ceramic restorations. Dent Mater. 2005;21(10):984-991.

3. Raigrodski AJ. Contemporary all-ceramic fixed partial dentures: a review. Dent Clin North Am. 2004;48(2):531-544.

4. Scherrer SS, De Rijk WG, Belser UC. Fracture resistance of human enamel and three all-ceramic crown systems on extracted teeth. Int J Prosthodont. 1996;9(6):580-585.

5. Albert FE, El-Mowafy OM. Marginal adaptation and microleakage of Procera AllCeram crowns with four cements. Int J Prosthodont. 2004;17(5):529-535.

6. Blatz MB. Long-term clinical success of all-ceramic posterior restorations. Quintessence Int. 2002;33(6):415-426.

7. Borges GA, Sophr AM, de Goes MF, Sobrinho LC, Chan DC. Effect of etching and airborne particle abrasion on the microstructure of different dental ceramics. J Prosthet Dent. 2003;89(5):479-488.

8. Gupta TK. Strengthening by surface damage in metastable tetragonal zirconia. J Am Ceram Soc. 1980;63(1-2):117.

9. Green DJ. A technique for introducing surface compression into zirconia ceramics. J Am Ceram Soc. 1983;66(10):c178-c179.

10. $\mathrm{Xu} \mathrm{HH}$, Jahanmir S, Ives LK. Effect of grinding on strength of tetragonal zirconia and zirconia-toughened alumina. Mach Sci Technol. 1997;1(1):49-66.

11. Kosmac T, Oblak C, Jevnikar P, Funduk N, Marion L. The effect of surface grinding and sandblasting on flexural strength and reliability of Y-TZP zirconia ceramic. Dent Mater. 1999;15(6):426-433.

12. Kosmač T, Oblak C, Jevnikar P, Funduk N, Marion L. Strength and reliability of surface treated Y-TZP dental ceramics. J Biomed Mater Res. 2000;53(4):304-313.

13. Aboushelib MN, Kleverlaan CJ, Feilzer AJ. Selective infiltrationetching technique for a strong and durable bond of resin cements to zirconia-based materials. J Prosthet Dent. 2007;98(5):379-388.

14. Kern M, Thompson VP. Sandblasting and silica coating of a glass-infiltrated alumina ceramic: volume loss, morphology, 
and changes in the surface composition. J Prosthet Dent. 1994; 71(5):453-461.

15. Zhang Y, Lawn BR, Rekow ED, Thompson VP. Effect of sandblasting on the long-term performance of dental ceramics. J Biomed Mater Res B Appl Biomater. 2004;71B(2):381-386.

16. Zhang $\mathrm{Y}$, Lawn BR, Malament KA, Van Thompson P, Rekow ED. Damage accumulation and fatigue life of particle-abraded ceramics. Int J Prosthodont. 2006;19(5):442-448.

17. Kosmac T, Wagner R, Claussen N. Determination of transformation depths in ceramics containing tetragonal $\mathrm{ZrO}$. J Am Ceram Soc. 1981;64(4):c-72-c-73.

18. Swain MV, Hannink RH. Metastablility of the martensitic transformation in a 12 mol\% ceriazirconia alloy: II, grinding studies. J Am Ceram Soc. 1989;72(8):1358-1364.

19. Özcan M, Vallittu PK. Effect of surface conditioning methods on the bond strength of luting cement to ceramics. Dent Mater. 2003;19(8):725-731.

20. Ritter JE, Bandyopadhyay N, Jakus K. Statistical reproducibility of dynamic and static fatigue experiments. Am Ceram Soc Bull. 1981;60(8):798-806.

21. Toraya H, Yoshimura M, Somiya S. Calibration curve for quantitative analysis of the monoclinic-tetragonal $\mathrm{ZrO}_{2}$ system by x-ray diffraction. J Am Ceram Soc. 1984;67(6):C-119-C-121.

22. Xible AA, de Jesus Tavarez RR, de Araujo CR, Bonachela WC. Effect of silica coating and silanization on flexural and composite -resin bond strengths of zirconia posts: An in vitro study. J Prosthet Dent. 2006;95(3):224-229.

23. ANSI/ADA Specification No. 69. Dental ceramic: 1999. Chicago: American Dental Association; 1999.

24. Souza ROA, Valandro LF, Melo RM, Machado JPB, Bottino MA, Özcan M. Air-particle abrasion on zirconia ceramic using different protocols: effects on biaxial flexural strength after cyclic loading, phase transformation and surface topography. J Mech Behav Biomed Mater. 2013;26:155-163.

25. Özcan M, Melo RM, Souza ROA, Machado JPB, Felipe Valandro L, Botttino MA. Effect of air-particle abrasion protocols on the biaxial flexural strength, surface characteristics and phase transformation of zirconia after cyclic loading. J Mech Behav Biomed Mater. 2013;20:19-28.

26. Sarmento HR, Campos F, Sousa RS, et al. Influence of airparticle deposition protocols on the surface topography and adhesion of resin cement to zirconia. Acta Odontol Scand. 2014;72(5):346-353.

27. Guazzato M, Quach L, Albakry M, Swain MV. Influence of surface and heat treatments on the flexural strength of Y-TZP dental ceramic. J Dent. 2005;33(1):9-18.

28. Piconi C, Maccauro G. Zirconia as a ceramic biomaterial. Biomaterials. 1999;20(1):1-25.

29. Chevalier J. What future for zirconia as a biomaterial? Biomaterials. 2006;27(4):535-543. 